OPEN ACCESS

Edited by:

Ovidiu Constantin Baltatu,

Anhembi Morumbi University, Brazil

Reviewed by:

Chang Hwa Joo,

Honam University, South Korea

Airong Qian,

Northwestern Polytechnical University,

China

*Correspondence:

Hojun Lee

hojun612@snu.ac.kr

Wook Song

songw3@snu.ac.kr

Specialty section:

This article was submitted to Integrative Physiology, a section of the journal

Frontiers in Physiology

Received: 26 January 2018

Accepted: 23 July 2018

Published: 13 August 2018

Citation:

Song $H$, Cho S, Lee $H-Y$, Lee $H$ and

Song W (2018) The Effects

of Progressive Resistance Exercise on Recovery Rate of Bone and Muscle in a Rodent Model of Hindlimb

Suspension. Front. Physiol. 9:1085. doi: 10.3389/fphys.2018.01085

\section{The Effects of Progressive Resistance Exercise on Recovery Rate of Bone and Muscle in a Rodent Model of Hindlimb Suspension}

\author{
Hansol Song', Suhan Cho', Ho-Young Lee ${ }^{2}$, Hojun Lee ${ }^{3,4 *}$ and Wook Song ${ }^{1,5 *}$ \\ 1 Health and Exercise Science Laboratory, Institute of Sport Science, Seoul National University, Seoul, South Korea, \\ ${ }^{2}$ Department of Nuclear Medicine, Seoul National University Bundang Hospital, Seongnam, South Korea, ${ }^{3}$ Department \\ of Rehabilitation Medicine, Seoul National University Bundang Hospital, Seongnam, South Korea, ${ }^{4}$ Department of Sports \\ and Health Science, Kyungsung University, Busan, South Korea, ${ }^{5}$ Institute on Aging, Seoul National University, Seoul, \\ South Korea
}

Purpose: This study aimed to examine the exercise-mediated musculoskeletal recovery following hindlimb suspension (HS) in order to identify whether bone modeling and muscle hypertrophy would eventuate in a synchronized manner during recovery stage.

Methods: To identify whether 2-week HS would be sufficient to induce a significant reduction of physiological indices in both tibia and adjacent hindlimb muscles, a total of 20 rats was randomized into 2-week HS $(n=10)$ and age-matched control group ( $n=10, \mathrm{CON})$. Another batch of rats were randomly assigned to three different groups to identify recovery intervention effects following suspension: (1) 2-week HS followed by 4-week spontaneous reloading recovery (HRE, $n=7$ ). (2) 2-week HS followed by 4-week progressive resistance ladder climbing exercise (HEX, $n=7)$. (3) Age-matched control (CON, $n=7)$. DXA, micro-CT, and ${ }^{18} \mathrm{~F}$-sodium fluoride (NaF) imaging, and EIA analysis were utilized to measure tibia bone indices. Hindlimb muscle wet weight and grip strength were measured to evaluate muscle mass and strength, respectively.

Results: In study 1, bone quality values [bone volume/total volume (BV/TV): $-27 \%$, areal bone mineral density (aBMD): $-23 \%$, mineral contents: $-7.9 \%$, mineral density: $-4.1 \%$, and bone density: $-38.9 \%]$ and skeletal muscle weight (soleus: $-46.8 \%$, gastrocnemius: $-19.6 \%$, plantaris: $-20.8 \%$, TA: $-22.8 \%$, and EDL: $-9.9 \%)$ were significantly lower in HS group compared to CON group. In study 2, micro-CT and DXAbased bone morphology (bone density, BT/TV, and aBMD) were fully recovered in HRE or HEX group. However, suspension-induced dysregulation of bone mineral metabolism was returned to age-matched control group in only HEX group, but not in HRE group. A greater level of biomarkers of bone formation (P1NF) and resorption (CTX-1) was observed in only HRE group compared to CON. The hindlimb skeletal muscle mass was significantly lower in both HRE and HEX groups compared to CON group. Hindlimb grip strength was the greatest in HEX group, followed by CON and HRE groups. 
Conclusion: Following HS, progressive resistance exercise promotes recovery rates of bone and skeletal muscle strength without a significant increase in muscular mass, suggesting that exercise-induced reacquisition of bone and muscle strength is independent of muscle hypertrophy during early recovery stage.

Keywords: skeletal muscle, bone, resistance exercise, hindlimb suspension, recovery

\section{INTRODUCTION}

Microstructure in bone tissue is constantly remodeled through the orchestrated actions of osteoclasts, osteoblasts, and osteocytes (Florencio-Silva et al., 2015). This dynamic process in conjunction with changes in muscle size is mainly modulated by repetitive mechanical resistance (Florencio-Silva et al., 2015). The musculoskeletal system is of critical importance in that it governs every movement of human body (Cointry et al., 2004). Since locomotion is mainly generated by bone's ability that endure mechanical force induced by the contraction of adjacent skeletal muscles, it is considered that bone and skeletal muscle develop and decline in a similar fashion over the course of life (Cianferotti and Brandi, 2014). In line with this, accumulating evidence suggested that there are potential cellular interplays between the two organs (Brotto and Johnson, 2014). A promising theory has suggested that osteocyte senses muscle contraction-derived mechanical resistance and converts strains into biochemical signals, thus regulating bone modeling (Bonewald, 2011; Nakashima, 2015). Although molecular link that tunes bone and muscle seems to be evident (DiGirolamo et al., 2013), it is uncertain as to whether the recovery of both organs is in a complete synchronism during regeneration stage.

The adaptability of human bone has been well demonstrated in weightlessness environment. Microgravity during spaceflight induces bone loss at a rate of $0.5-1.5 \%$ a month (Lang et al., 2004). Such a loss tends to be persistent over 2.5 years following a stay on International Space Station (ISS) (Dana Carpenter et al., 2010; Leblanc et al., 2013). In addition to loss of bone quality, microgravity-induced muscle atrophy is evident. Astronauts subjected to a 17 -day microgravity experienced an $8 \%$ decline in cross-sectional area of skeletal muscle (Tesch et al., 2005), thereby suggesting a reduction of bone and skeletal muscle under microgravity condition. Although human-based microgravity studies elicit direct clinical application, there is an extremely limited human-based data set due to the cost of performing largescale experiment (Lloyd et al., 2014). A need for ground-based experiment of microgravity led to the development of a rodent hindlimb suspension (HS) model (Morey-Holton and Globus, 2002). Multiple studies demonstrated a potent reproducibility of HS-induced skeletal muscle atrophy and bone loss (Grano et al., 2002; Hurst and Fitts, 2003; Pan et al., 2008). Specifically, bone mass, density, mineralization, trabecular thickness, and osteoblastic activity are negatively affected following the chronic unloading treatment (Roer and Dillaman, 1990; Dehority et al., 1999; Bloomfield et al., 2002; Globus et al., 2015). In addition, bone loss with architectural disarray, tissue deterioration, and osteopenia are induced due to the suspension-induced absence of mechanical loading (Ohira et al., 2006).
Under spontaneous re-ambulation condition, experimental rodents fail to recover HS-induced loss of mineral content and bone formation rate during a period of recovery greater than the unloading duration, thereby suggesting that spontaneous normal weight bearing is not sufficient to induce fully recovered bone condition (Vico et al., 1995; Sakata et al., 1999). As compared with bone, suspension-induced reduction of muscle contractile properties has been documented to recover to pre-suspension level at a faster rate (Warren et al., 2004; Allen et al., 2006). The temporal difference of both organs' recovery rate has been considered to induce a physiological mismatch in the production of force, thus increasing a possibility of musculoskeletal injury during rehabilitation (Allen et al., 2006). Several interventions have gained attention as countermeasures that preserve muscle and bone during or following a period of unloading (Ju et al., 2013; Jang et al., 2016). Among them, resistance exercise has been proven to be an effective strategy to accelerate the recovery rate of both organs. However, to the best of our knowledge, it has not been explored whether exercise-mediated recovery of both organs would occur in a complete simultaneity following HS.

Therefore, the purposes of this study were to investigate exercise and spontaneous reambulation-mediated recovery rate of tibia and related hindlimb muscles following HS. To achieve these aims, two related studies were performed. In study 1 , 9-week-old SD rats were hindlimb-suspended for 2 weeks in order to confirm that 2-week HS would induce sufficient reduction of both muscle and bone. After the confirmation of our HS protocol, study 2 was performed by assigning second round of age-matched rats to each of the following three groups: (1) HS for 2 weeks followed by 4 weeks of spontaneous reloading recovery (HRE). (2) HS for 2 weeks followed by 4 weeks of progressive resistance ladder climbing exercise (HEX). (3) Another group of rats were housed in cages without HS during 6 weeks as an age-matched control (CON). We hypothesized that progressive resistance exercise would alter recovery rate of bone and/or muscle, as compared with weight bearing spontaneous recovery.

\section{MATERIALS AND METHODS}

\section{Animal Protocol}

Sprague-Dawley rats (Orient Bio Co., Korea) were used in the study. All the rats were 9-week-old and were housed in a controlled environment in $12: 12$-h light-dark cycle at $22^{\circ} \mathrm{C}$. The rats were fed with water and food (Rodent NIH-31 Open Formula Auto, Zeigler Bros Inc., United States) ad libitum. Experiments were approved by the Institutional Animal Care and Use Committee (IACUC) of Seoul National University. The IACUC number is SNU-150820-4-4. One week after arrival, first 
batch of rats were randomly assigned to either cage: control (CON, $n=10)$ or HS groups $(n=10)$ (Figure 1). In HS group, rats were taped on tails for HS for 2 weeks. Specifically, the rats were tail-suspended to the installed metal harness which holds rats in hindlimb suspended position by connecting taped tail to the pulley. Rats in control groups were housed in standard cages without access to metal harness for 2 weeks. They were used as an age-matched control against HS. After confirming whether 2-week HS protocol induced sufficient reduction of both bone and muscle, additional batch of rats were used to investigate the effects of spontaneous reloading and progressive resistance exercise on muscle and bone recovery following HS. Specifically, 1 week after arrival, another batch of rats were randomly assigned to three different groups: HS for 2 weeks followed by 4 weeks of spontaneous reloading recovery (HRE, $n=7$ ), HS for 2 weeks followed by 4 weeks of progressive resistance ladder climbing exercise (HEX, $n=7$ ). The rats in cages without any intervention were used as an age-matched control (CON, $n=7)$.

\section{Hindlimb Suspension Protocol}

In order to make rat's hindlimb to be suspended, taping was performed to attach rat tails to metal harness located above the ground surface. Two rats were housed in a HS cage (length $60 \mathrm{~cm} \times$ width $-30 \mathrm{~cm} \times$ height $-40 \mathrm{~cm}$ ) and were separated by an opaque barricade in order to eliminate any interaction between rats. Each rat was allowed to roam freely around in its own space $(30 \mathrm{~cm} \times 30 \mathrm{~cm})$ by attaching their tail to a pulley allowing a $360^{\circ}$ spin. The height of the taping was continuously adjusted according to a rat's size in order for rats to stay in fully hindlimb suspended position. Grip strength was measured before and after intervention. The physical characteristics of the rats are presented in Table 2.

\section{Exercise Protocol}

Progressive ladder climbing exercise was performed in HEX group following 2 weeks of HS treatment. The rats were subjected to a programmed exercise protocol 3 days a week based on a previous study (Westerlind et al., 1998). Exercise intervention was implemented on a 1-m ladder with $2-\mathrm{cm}$ grid and $0.25-\mathrm{m}$ width at $85^{\circ}$ inclination against a wall. Since the rats' physical function had been declined due to suspension treatment, exercise intensity was carefully managed in a progressive manner. Specifically, prior to the first exercise session, 1-day recovery period was provided to the rats. No weight was attached to rat's tail at the start of exercise intervention. If the rats were unable to accomplish a certain weight load, previous weight load was used to encourage them to accomplish a total of 10 bouts of climbing. Only gentle brush to rats was used to encourage rats to accomplish each exercise bout. The detailed exercise protocol is presented in Table 1.

\section{Bone Quality Analysis - PET $/ \mu$ CT and Micro-CT}

In vivo $\mathrm{Na}^{18} \mathrm{~F}$ PET/CT (positron emission tomography/ computed tomography) scanning was performed to measure bone quality indices using a Nano PET/CT in vivo pre-clinical imager (Mediso, Hungary). Scanning was performed according to the protocol and procedure in the department of nuclear medicine in Seoul National University Bundang Hospital. Dynamic PET studies were performed after the intravenous application of ${ }^{18}$ F-fluoride. A dedicated PET-CT system with an

TABLE 1 | Progressive resistance exercise protocol.

\begin{tabular}{lcccc}
\hline & Week 1 & Week 2 & Week 3 & Week 4 \\
\hline Repetition & \multicolumn{4}{c}{ 10 repetition } \\
\% load/BW & $0-50$ & 60 & 70 & 80 \\
Rest & \multicolumn{4}{c}{ 2 min interval between repetition } \\
Frequency & \multicolumn{3}{c}{ 3 sessions per week }
\end{tabular}

Progressive resistance ladder climbing exercise was performed in HEX group following 2 weeks of hindlimb suspension. Exercise intervention was implemented on a 1-m ladder with 2-cm grid and $0.25-\mathrm{m}$ width at $85^{\circ}$ inclination against a wall. Gentle brush to rats was used to encourage rats to accomplish each exercise bout. If the rats were unable to accomplish a certain weight load, previous weight load was used to encourage them to accomplish a total of 10 bouts.

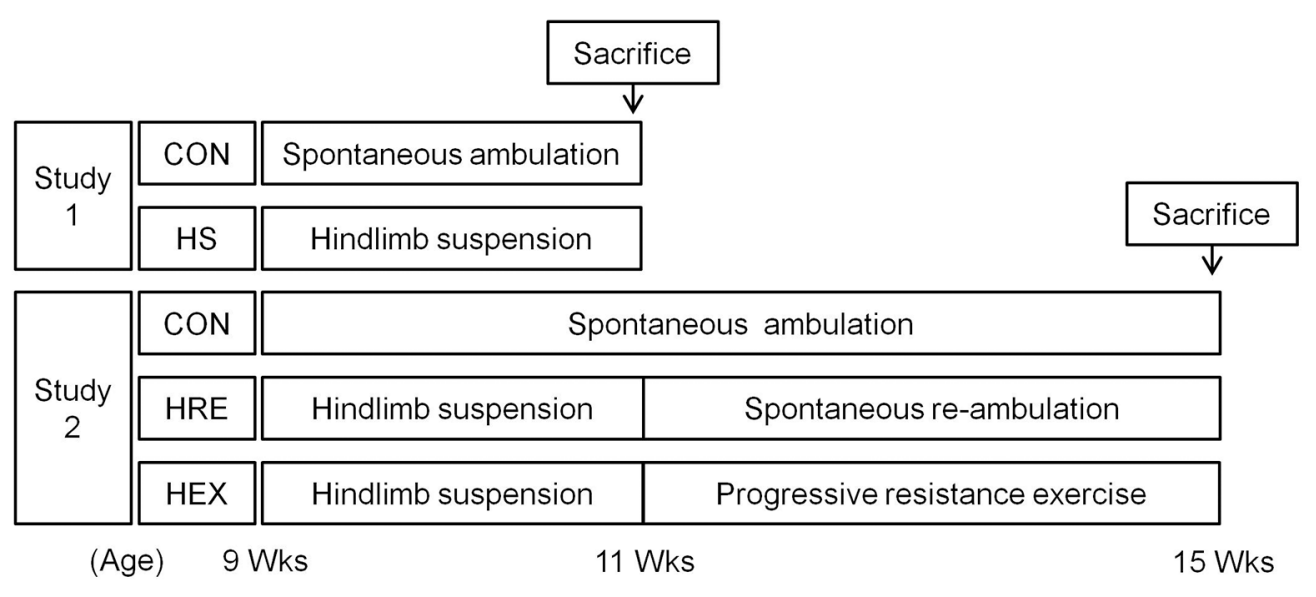

FIGURE 1 | Schematic diagram of study design. 
axial field of view was operated in a three-dimensional mode and was used for all animals. The system provides the simultaneous acquisition of transverse slices with a slice thickness of $0.6 \mathrm{~mm}$. The animals were positioned in the axial plane of the system to acquire the best resolution in the middle of the system. CT scan was performed prior to the PET scanning. Bone density was analyzed in Hounsfield Unit (HU) and trabecular BV/TV (bone volume/total volume) in percent (\%). Each result was analyzed distinctively. All rats were anesthetized with isoflurane gas inhalation prior to scanning.

\section{Body Composition and Bone Mineral Density - DXA}

In vivo DXA (Dual energy X-ray Absorptiometry) scanning was performed to measure body composition, whole body and areal bone mineral density ( $\mathrm{BBMD})$, and contents of rats using a Hologic Discovery W model (Hologic, United States). aBMD and areal bone mineral contents (aBMC) were taken from tibia of rats. For body composition indices, the values of fat mass, lean mass, and $\%$ fat were used for this study. All rats were anesthetized for DXA scanning and sacrifice was performed subsequently.

\section{Serum Bone Turnover Marker Analysis - EIA Analysis}

The measurement of bone turnover markers in serum was performed by enzyme immunoassay (EIA) method. Procollagen of type I collagen (P1NP) was selected as a bone formation marker (Rat/Mouse P1NP EIA, Immunodiagnostic Systems). C-terminal telopeptide (CTX-1) was selected as a bone resorption marker (RatLaps ${ }^{\text {TM }}$ CTX-1 EIA, Immunodiagnostic Systems). The sensitivity of P1NP in the analysis was $0.7 \mathrm{ng} / \mathrm{mL}$, and the inter-assay and intra-assay CV were 9.2-8.2 and 6.4-5.0\%. The CTX-1 RatLaps ${ }^{\text {TM }}$ detection limit was $2.0 \mathrm{ng} / \mathrm{mL}$, and the interassay and intra-assay coefficients of variation were 9.2-5.8 and $14.8-10.7 \%$, respectively.

\section{Grip Strength}

The grip strength was measured using Grip Strength Meter (Bioseb, France). The test was performed in rat's hindlimb by allowing the animals to grasp a bar attached to the force gauge, followed by pulling the animal away from the gauge in a progressive manner. Specifically, rats were positioned by allowing it to put its forelimb onto the researcher's left hand to avoid any interference of its forelimb to the gauge. The test was performed five times in each hindlimb.

\section{Skeletal Muscle Collection}

For skeletal muscle dissection, an incision was made through the skin around ankle area and the skin was reflected to expose the muscles of the lower leg. The Achilles tendon was cut to remove soleus, plantaris, and gastrocnemius. To dissect tibialis anterior and extensor distorum longus, the fascia covering the muscles was carefully removed and extensor ligament was cut to release the distal tendon and use it to peel them off with a fine surgical scissors. The dissected muscles were cleaned of excess fat and external connective tissue before the measurement.

\section{Statistical Analysis}

Statistical analysis was performed using SPSS 22.0 software. Results were expressed as mean \pm SEM. T-test was performed to examine the difference between two groups in study 1. Oneway ANOVA was performed to examine the difference in study 2 , followed by Bonferroni's post hoc test. The level of significance was set at $p<0.05$.

\section{RESULTS}

\section{The Effects of a 2-Week Hindlimb Suspension on Body Composition}

Hindlimb suspension of experimental rats for 14 days resulted in significant changes in body composition (Table 2). Total mass $(-11.3 \%)$ and lean mass $(-15.2 \%)$ were significantly decreased after 14 days of HS compared to age-matched controls. Although total fat mass was not changed statistically, body fat $(38.1 \%)$ was significantly increased by HS.

\section{The Effects of a 2-Week Hindlimb Suspension on Bone and Muscle}

Hindlimb suspension led to significant changes in tibia and four different hindlimb muscles (Figure 2 and Table 3). Specifically, tibia bone density $(-33.8 \%)$, BT/AT $(-37.3 \%)$, aBMD $(-33.2 \%)$, bone mineral contents $(-7.5 \%)$, and bone mineral density $(-4.1 \%)$ were decreased significantly after HS. Serum P1NP (biomarker of bone formation) levels were significantly lower in HS group compared to CON group $(p<0.009)$. Serum CTX-1 (biomarker of bone resorption) level was not different between two groups. The wet weight of four different skeletal muscles adjacent to tibia was measured during surgical procedure. Soleus $(-46.8 \%)$, gastrocnemius $(-19.6 \%)$, tibialis anterior $(-12.1 \%)$, extensor distorum longus $(-9.9 \%)$, and plantaris $(-19.8 \%)$ were decreased significantly in HS group compared to age-matched control.

\section{Spontaneous Ambulation and Resistance Exercise-Mediated Bone Turnover Rate Following Hindlimb Suspension}

The $\mathrm{Na}^{18} \mathrm{~F}$ PET/CT scan was performed and the lower portion of rats were focused for the tibia metaphysis analysis (Figure 3).

TABLE 2 | Physical characteristics after hindlimb suspension.

\begin{tabular}{lrl}
\hline & CON $(\boldsymbol{n}=\mathbf{1 0})$ & Hindlimb suspension $(\boldsymbol{n}=\mathbf{1 0})$ \\
\hline Total mass $(\mathrm{g})$ & $335.250 \pm 3.918$ & \multicolumn{1}{c}{$301.690 \pm 7.339^{* *}$} \\
Lean mass $(\mathrm{g})$ & $288.190 \pm 4.071$ & $250.330 \pm 6.137^{* * *}$ \\
Fat mass $(\mathrm{g})$ & $36.290 \pm 2.425$ & $41.420 \pm 1.754$ \\
Fat $(\%)$ & $10.840 \pm 0.718$ & $13.800 \pm 0.453^{* *}$ \\
\hline
\end{tabular}

Lean mass, fat mass, and percent fat were measured by DXA analysis following 2-week hindlimb suspension $(n=10)$. Age-matched rats were used as control $(n=10)$. All the results were presented as mean $\pm S E M,{ }^{* *} p<0.01$ vs. CON, *** $p<0.001$ vs. CON. 


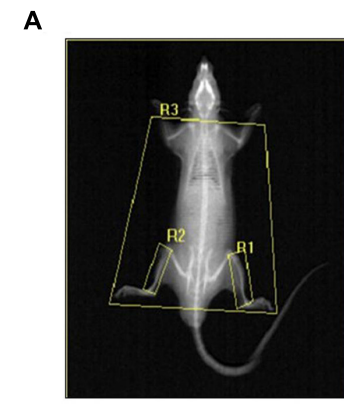

C

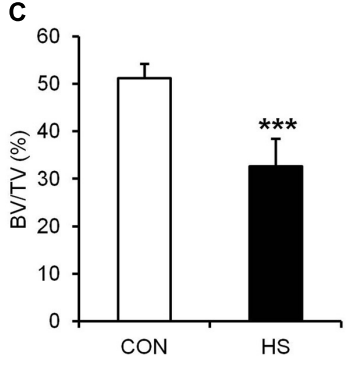

$\mathbf{F}$

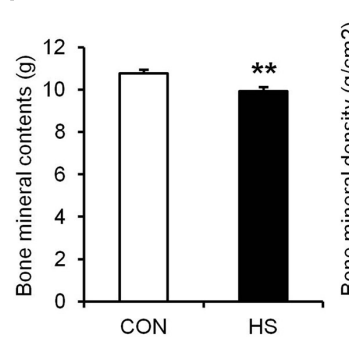

B

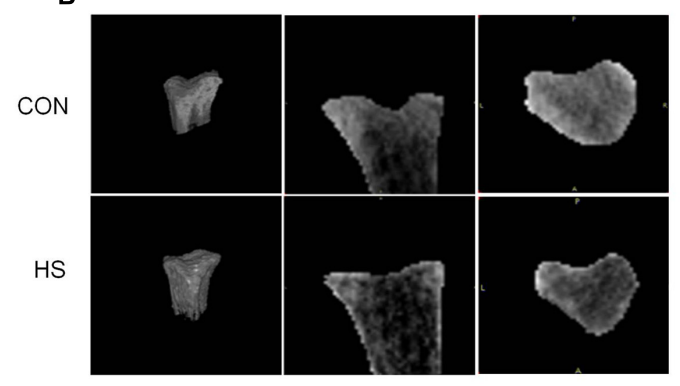

D

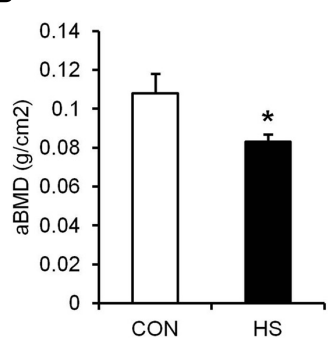

E

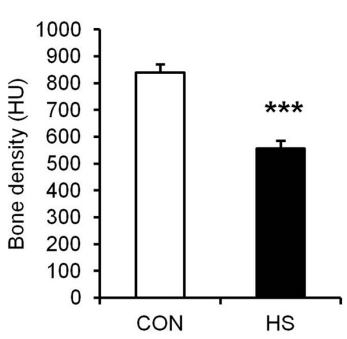

G

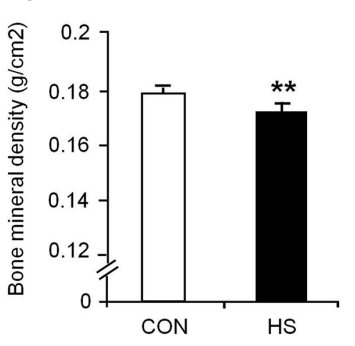

H

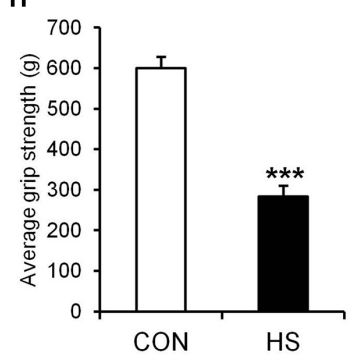

FIGURE 2 | The effects of hindlimb suspension on bone morphology and grip strength. (A) Image of ROI area selection of DXA scan (R1, right tibia; R2, left tibia; R3, whole body without head/global: whole body). (B) Representative micro-CT images of animals' tibiae. (C) BV/TV, (D) aBMD, (E) bone density, (F) bone mineral contents, (G) bone mineral density, and (H) hindlimb grip strength were measured after 2 -week hindlimb suspension. ${ }^{*} p<0.05$ vs. CON, ${ }^{* *} p<0.01$ vs. CON, ${ }^{* * *} p<0.001$ vs. CON. All the results were presented as mean $\pm \mathrm{SE}$.

The result shows that mineral metabolism (bone turnover rate) of both sides of tibia was significantly higher in HRE group compared to CON and HEX groups $(p<0.05)$. Serum levels of P1NP (biomarker of bone formation) and CTX-1 (biomarker of bone resorption) were also significantly higher in HRE group compared to CON and HEX groups $(p<0.05)$.

TABLE 3 | Skeletal muscles weight following hindlimb suspension.

\begin{tabular}{lcc}
\hline & CON $(\boldsymbol{n}=\mathbf{1 0})$ & Hindlimb suspension $(\boldsymbol{n}=\mathbf{1 0})$ \\
\hline Soleus $(\mathrm{g})$ & $0.265 \pm 0.011$ & $0.141 \pm 0.007^{* * *}$ \\
Gastrocnemius $(\mathrm{g})$ & $3.383 \pm 0.054$ & $2.722 \pm 0.071^{* * *}$ \\
TA (g) & $1.242 \pm 0.018$ & $1.092 \pm 0.033^{* *}$ \\
EDL (g) & $0.3178 \pm 0.0116$ & $0.2863 \pm 0.0061^{*}$ \\
Plantaris (g) & $0.6799 \pm 0.0164$ & $0.5385 \pm 0.0179^{*}$
\end{tabular}

Skeletal muscles adjacent to tibia were weighted following 2-week hindlimb suspension $n=10)$. Age-matched rats were used as control $(n=10)$. TA, tibialis anterior; EDL, extensor digitorum longus. All the results were presented as mean \pm SEM, ${ }^{*} p<0.05$ vs. CON, ${ }^{* *} p<0.01$ vs. CON, ${ }^{* *} p<0.001$ vs. CON.

\section{Spontaneous Ambulation and Resistance Exercise-Mediated Bone Qualities Following Hindlimb Suspension}

Tibia-specific regional micro-CT scanning was performed to obtain three-dimensional and cross sectional images of tibia for qualitative analysis (Figure 4). Bone density was analyzed in $\mathrm{HU}$ and trabecular BV/TV in percent. Bone density $(p<0.05)$, $\mathrm{BV} / \mathrm{TV}(p<0.01)$, and aBMD $(p<0.05)$ were significantly higher in HEX group compared to CON and HRE groups. There was no difference observed between CON and HRE groups.

\section{Spontaneous Ambulation and Resistance Exercise-Mediated Muscle Weight Following Hindlimb Suspension}

Four different skeletal muscles adjacent to tibia were weighed during surgical procedure (Table 4). The weights of soleus $(p<0.01)$, gastrocnemius, tibialis anterior, EDL, and plantaris $(p<0.05)$ were significantly lower in HRE and HEX groups compared to age-matched control. However, these muscles' 
A

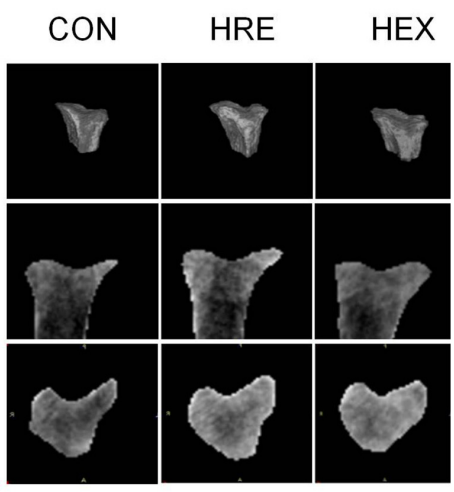

C

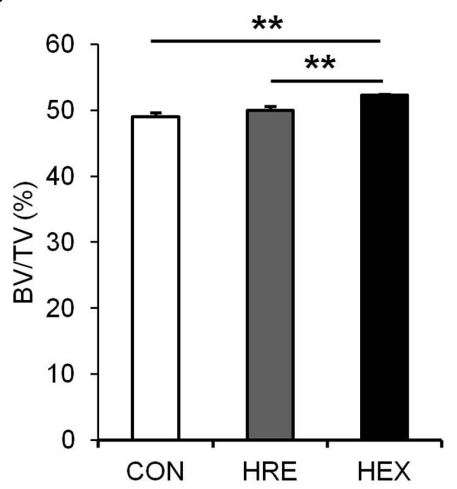

B

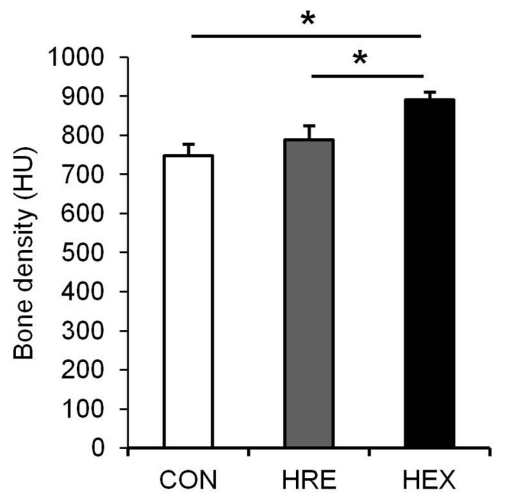

D

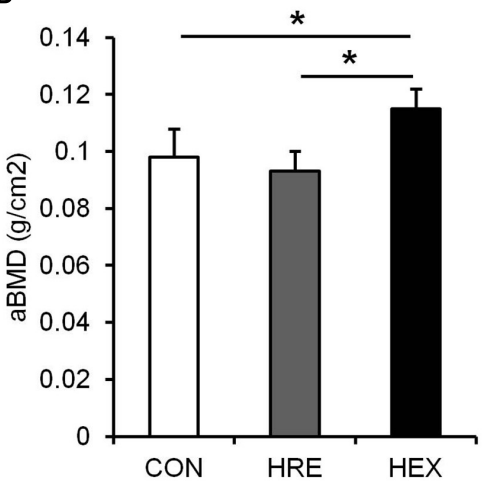

FIGURE 3 | The effects of re-ambulation and exercise on bone morphology following hindlimb suspension. (A) Representative micro-CT images of animals' tibiae. (B) Bone density, (C) BV/TV, and (D) aBMD were measured after 4-week intervention. ${ }^{*} p<0.05,{ }^{* *} p<0.01$. All the results were presented as mean $\pm \mathrm{SE}$.

weight was not statistically different between HRE and HEX groups.

\section{Hindlimb Grip Strength Before and After Spontaneous Ambulation and Resistance Exercise}

The grip strength was calculated by the average of five records of each rat. In study 1 (Figure $\mathbf{2 H}$ ), grip strength was significantly lower in HS group compared to CON $(p<0.05)$. In study 2 (Figure 5), grip strength was lower in HRE and HEX groups compared to age-matched control before recovery intervention $(p<0.05)$. Following recovery intervention, grip strength was the highest in HEX group, followed by CON and HRE $(p<0.05)$.

\section{DISCUSSION}

The main purpose of this study was to investigate progressive resistance exercise-mediated recovery indices of bone and skeletal muscles following 2-week HS. Our results clearly showed that 2week HS induces a significant reduction of physiological indices in both tibia and adjacent hindlimb muscles. Interestingly, progressive resistance exercise promoted recovery rates of tibia and hindlimb muscular strength in the absence of an increase of related muscle mass.

\section{The Effects of Hindlimb Suspension on Bone and Muscles}

Hindlimb suspension is a well-documented model as groundbased analog to identify the effects of unloading condition on musculoskeletal system, thereby resulting in concomitant loss of both muscle and bone (Morey-Holton et al., 2005; Colaianni et al., 2017). In accordance with previous findings (Swift et al., 2010; Jing et al., 2014), our results revealed significant deterioration of both tissues after only 2 weeks of suspension (Figure 2 and Table 3), validating our HS protocol. In the study, the weights of five major skeletal muscles (soleus, gastrocnemius, tibialis anterior, EDL, and plantaris) were measured due to their anatomical proximity to tibia. Interestingly, the reduction of individual muscle weight was determined to be in a broad spectrum (soleus: $-46.8 \%$, gastrocnemius: $-19.6 \%$, tibialis anterior: $-12.1 \%$, EDL: $-9.9 \%$, and plantaris: $-19.8 \%$ ). This implies a possibility that microgravity-simulated atrophy is induced in a muscle typedependent manner. This idea is further supported by several lines of studies indicating that the loss of protein was more dramatic in slow-twitch muscles than fast-twitch muscles (Tsika et al., 1987; Hornberger et al., 2001; Wang et al., 2017). Based on the fact that soleus muscle presents a predominance of slow twitch type I fibers while the other four muscles consist of mainly fast twitch type II fibers or a mixture (Cornachione 


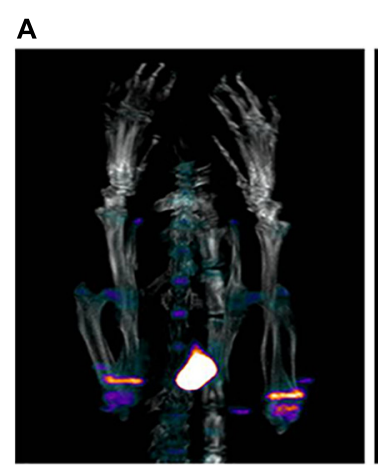

CON

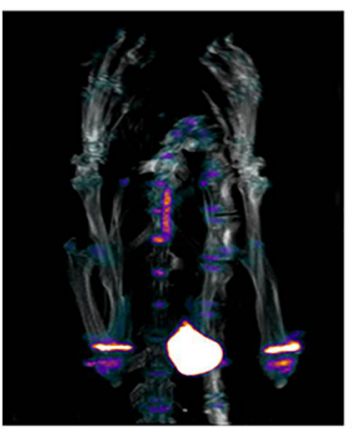

HRE

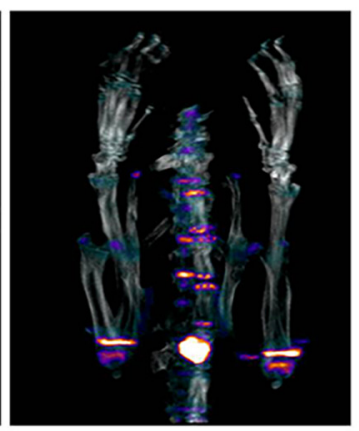

HEX
B

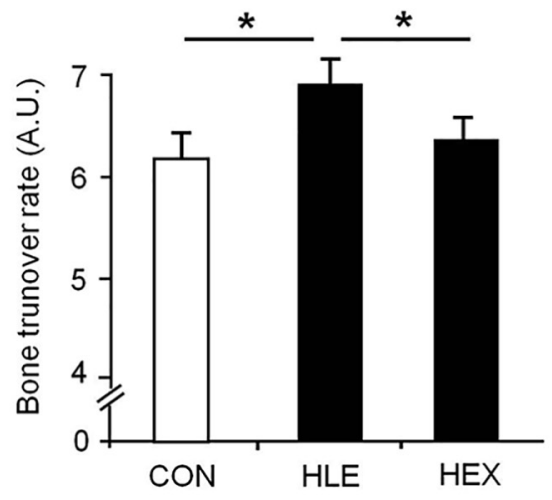

D

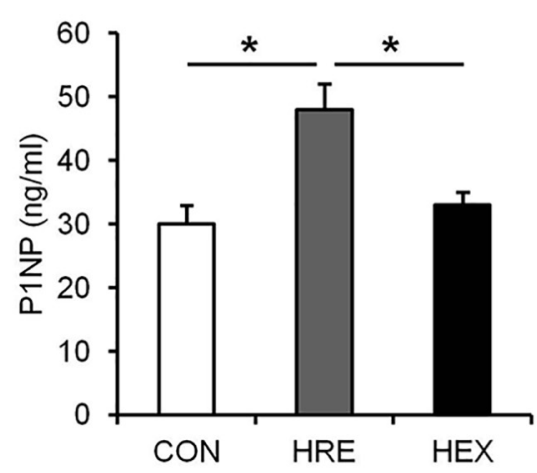

C

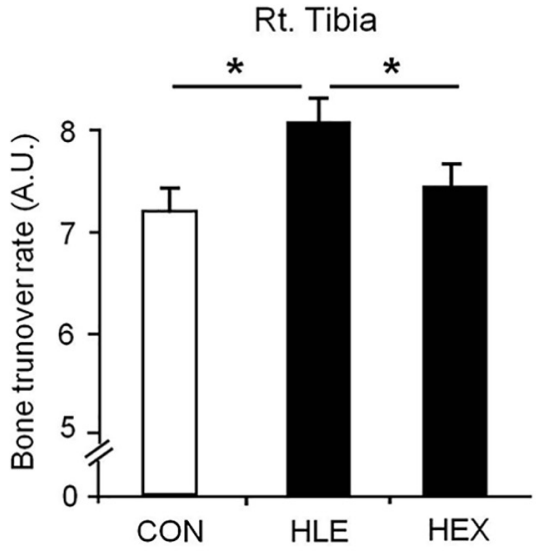

E

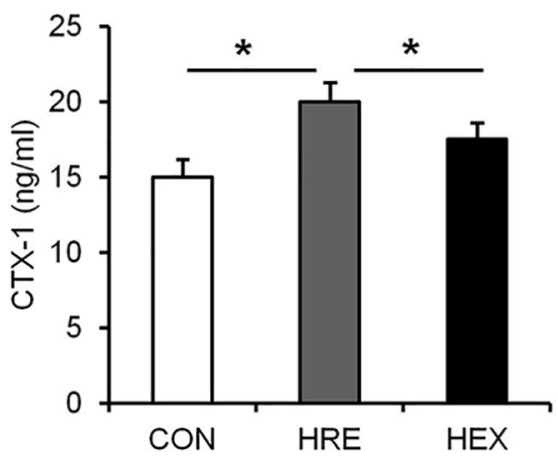

FIGURE 4 | The effects of re-ambulation and exercise on bone mineral metabolism following hindlimb suspension. (A) Representative PET/CT fusion images of tibia metaphysis. Bone turnover rate of (B) left and (C) right tibia was obtained from PET/CT fusion images. Biomarkers of (D) bone formation (P1NP) and (E) bone resorption (CTX-1) were measured by EIA analysis. ${ }^{*} p<0.05$. All the results were presented as mean $\pm \mathrm{SE}$.

et al., 2011), it is considered that the different atrophy rates are attributed to the different fiber type composition in each muscle.

The muscle atrophies concomitant with the suspensionmediated bone loss (bone density, BV/TV, aBMD, bone mineral contents, and bone mineral density) indicates that 2-week hindlimb suspension is sufficient to induce a significant deterioration of musculoskeletal system.

\section{The Effects of Spontaneous}

\section{Reambulation and Resistance Exercise} on the Recovery of Bone and Muscles

A variety of approaches have been implemented to counteract the loss of bone and skeletal muscles. Although exercise is considered as an efficient and practical strategy to facilitate the recovery of both tissues, most of the related studies has been focused on 
TABLE 4 | Recovery intervention-mediated skeletal muscles weight following hindlimb suspension.

\begin{tabular}{lccc}
\hline & CON $(\boldsymbol{n}=\mathbf{7})$ & HRE $(\boldsymbol{n}=7)$ & HEX $(\boldsymbol{n}=7)$ \\
\hline Soleus $(\mathrm{g})$ & $0.4021 \pm 0.0181$ & $0.3692 \pm 0.0129^{* *}$ & $0.3539 \pm 0.0093^{* *}$ \\
Gastrocnemius (g) & $4.9505 \pm 0.1365$ & $4.6156 \pm 0.0733^{*}$ & $4.5153 \pm 0.0741^{*}$ \\
Tibialis anterior (g) & $1.9068 \pm 0.0615$ & $1.7592 \pm 0.0363^{*}$ & $1.6978 \pm 0.0482^{*}$ \\
EDL (g) & $0.4643 \pm 0.0246$ & $0.4297 \pm 0.0078^{*}$ & $0.4224 \pm 0.0054^{*}$ \\
Plantaris (g) & $0.9712 \pm 0.0348$ & $0.9396 \pm 0.0179^{*}$ & $0.9271 \pm 0.0196 *$
\end{tabular}

Skeletal muscles adjacent to tibia were weighted following 4-week recovery intervention ( $n=7)$. Age-matched rats were used as control $(n=7)$. TA, tibialis anterior; $E D L$, extensor digitorum longus. All the results were presented as mean \pm SEM, ${ }^{*} p<0.05$ vs. CON, ${ }^{* *} p<0.01$ vs. CON.

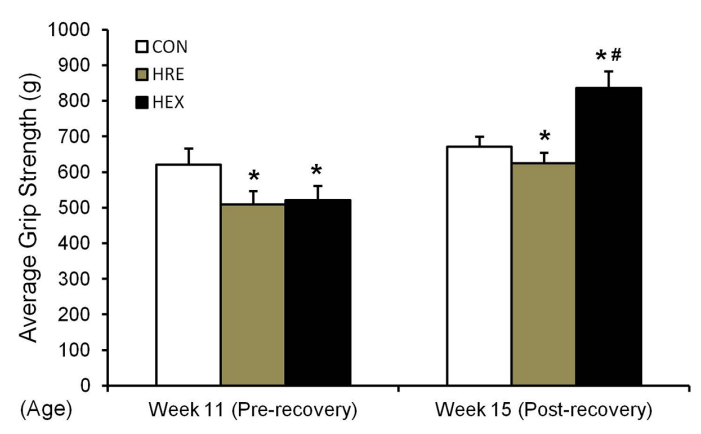

FIGURE 5 | Hindlimb grip strength before and after recovery intervention. The grip strength test was performed in rat's hindlimb by allowing the animals to grasp a bar attached to the force gauge, followed by pulling the animal away from the gauge in a progressive manner. The test was performed five times. ${ }^{*} p<0.05$ vs. CON, ${ }^{\#} p<0.05$ vs. HRE. All the results were presented as mean \pm SE.

either muscle or bone (Ju et al., 2013; Song et al., 2017), limiting a comprehensive understanding of physiological link between two tissues during recovery stage.

As our multi-faceted approaches demonstrated, a greater recovery of micro-CT (bone density and BV/TV) and DXAbased bone parameters (aBMD) was observed in HEX group compared to HRE group (Figure 3 ). These results indicate that morphological recovery of the bones was promoted by progressive resistance exercise, which are in line with a previous study indicating the therapeutic effect of exercise on bone (Shirazi-Fard et al., 2014). Bone is a dynamic tissue changing its strength and micro-morphologic features by regulating bone turnover rate via actions of osteoblasts and osteoclasts (van der Eerden et al., 2014). Weakness of bone strength and stiffness is the manifestation of excessive or deficient bone turnover rate. ${ }^{18} \mathrm{~F}$-sodium fluoride $(\mathrm{NaF})$ imaging has been proven to catch calcium metabolic activity in the bone structures, which could be easily unnoticed from analyzing the morphological images such as micro-CT and DXA (Jadvar et al., 2015). Thus, bone turnover rate (bone mineral metabolism) was measured using ${ }^{18} \mathrm{~F}-\mathrm{NaF}$ for further investigation. Interestingly, despite of complete recovery of the CT- and DXA-based bone morphology in HLE group compared to age-matched control, tibia mineral metabolism was abnormally elevated in HRE group compared to CON and HEX group (Figure 4), providing a piece of biochemical evidence that post-suspension bone modeling was not completed under the condition of spontaneous reambulation. In order to intensify the interpretation of the dysregulated bone turnover rate, serum P1NP (bone formation) and CTX-1 (bone resorption) were tested as surrogate markers of osteoblast and osteoclast activities. In agreement of our PET/CT data, these markers were significantly elevated in HRE group compared to both CON and HEX group (Figures 4D,E). Considering our experimental design of 2 -week HS and subsequent 4-week recovery intervention, it is considered that tibia mineral metabolism requires at least more than two times the duration of reambulation for the complete recovery. This idea is in agreement with a previous study that bone requires nearly three times the period of re-ambulation to return to age-matched control levels (Allen et al., 2006). In addition, with multi-faceted analyses of DXA, micro-CT, and ${ }^{18} \mathrm{~F}-\mathrm{NaF}$ imaging, the current study supports a notion that the duration of morphological and biochemical bone recovery could be significantly reduced by progressive resistance exercise (Russo, 2009; Shirazi-Fard et al., 2014).

In the study, five major muscles adjacent to tibia were weighed to exam whether progressive resistance exercise would induce the re-acquisition of muscle mass concomitant with improved bone qualities. Interestingly, all the muscles' weight was not different between HRE and HEX groups while the grip strength was the greatest in HEX group (Table 4 and Figure 5). The increased force production without regain of muscle mass implies a possibility of peripheral neural recovery-mediated force production under early regeneration phases. This idea is supported by a recent study on peripheral motor axons, indicating that suspension-induced reduction of a neuromuscular axonal excitability property returns to control range after a short duration of recovery (Banzrai et al., 2016). Thus, it is considered that progressive resistance exercise induces a faster recovery of peripheral nervous system compared to muscle hypertrophy to fine-tune force generation in order to prevent skeletal muscle dominance-induced fracture under the regeneration process.

\section{CONCLUSION}

Our multi-faceted study demonstrated that progressive resistance exercise following $\mathrm{HS}$ promotes recovery rates of bone morphology and skeletal muscle strength in the absence of a significant increase in muscle mass. This suggests that exercise-induced regain of muscular and bone strength is independent of muscle hypertrophy during early recovery stage. In future endeavor, a greater understanding of physiological link among muscle, bone, and neuroaxonal properties would provide additional insight into the development of promising strategies to counteract musculoskeletal and neuromuscular pathophysiological condition.

\section{AUTHOR CONTRIBUTIONS}

All authors listed have made a substantial and intellectual contribution to the work, and approved it for publication. HS 
contributed in the acquisition and analysis of data, and drafting the work. SC and H-YL contributed in the acquisition and analysis of data for the work. HL contributed in the interpretation of data for the work, revising the work critically, and final approval of the version to be published. WS contributed in the conception of the work, revising the work critically, and final approval of the version to be published.

\section{REFERENCES}

Allen, M. R., Hogan, H. A., and Bloomfield, S. A. (2006). Differential bone and muscle recovery following hindlimb unloading in skeletally mature male rats. J. Musculoskelet. Neuronal Interact. 6, 217-225.

Banzrai, C., Nodera, H., Kawarai, T., Higashi, S., Okada, R., Mori, A., et al. (2016). Impaired axonal $\mathrm{Na}(+)$ current by hindlimb unloading: implication for disuse neuromuscular atrophy. Front. Physiol. 7:36. doi: 10.3389/fphys.2016. 00036

Bloomfield, S. A., Allen, M. R., Hogan, H. A., and Delp, M. D. (2002). Site- and compartment-specific changes in bone with hindlimb unloading in mature adult rats. Bone 31, 149-157. doi: 10.1016/S8756-3282(02)00785-8

Bonewald, L. F. (2011). The amazing osteocyte. J. Bone Miner. Res. 26, 229-238. doi: $10.1002 /$ jbmr.320

Brotto, M., and Johnson, M. L. (2014). Endocrine crosstalk between muscle and bone. Curr. Osteoporos. Rep. 12, 135-141. doi: 10.1007/s11914-014-0209-0

Cianferotti, L., and Brandi, M. L. (2014). Muscle-bone interactions: basic and clinical aspects. Endocrine 45, 165-177. doi: 10.1007/s12020-013-0026-8

Cointry, G. R., Capozza, R. F., Negri, A. L., Roldan, E. J., and Ferretti, J. L. (2004). Biomechanical background for a noninvasive assessment of bone strength and muscle-bone interactions. J. Musculoskelet. Neuronal Interact. 4, 1-11.

Colaianni, G., Mongelli, T., Cuscito, C., Pignataro, P., Lippo, L., Spiro, G., et al. (2017). Irisin prevents and restores bone loss and muscle atrophy in hind-limb suspended mice. Sci. Rep. 7:2811. doi: 10.1038/s41598-017-02557-8

Cornachione, A. S., Benedini-Elias, P. C., Polizello, J. C., Carvalho, L. C., and Mattiello-Sverzut, A. C. (2011). Characterization of fiber types in different muscles of the hindlimb in female weanling and adult Wistar rats. Acta Histochem. Cytochem. 44, 43-50. doi: 10.1267/ahc.10031

Dana Carpenter, R., LeBlanc, A. D., Evans, H., Sibonga, J. D., and Lang, T. F. (2010). Long-term changes in the density and structure of the human hip and spine after long-duration spaceflight. Acta Astronaut. 67, 71-81. doi: 10.1016/j. actaastro.2010.01.022

Dehority, W., Halloran, B. P., Bikle, D. D., Curren, T., Kostenuik, P. J., Wronski, T. J., et al. (1999). Bone and hormonal changes induced by skeletal unloading in the mature male rat. Am. J. Physiol. 276(1 Pt 1), E62-E69. doi: 10.1152/ajpendo. 1999.276.1.E62

DiGirolamo, D. J., Kiel, D. P., and Esser, K. A. (2013). Bone and skeletal muscle: neighbors with close ties. J. Bone Miner. Res. 28, 1509-1518. doi: 10.1002/jbmr. 1969

Florencio-Silva, R., Sasso, G. R., Sasso-Cerri, E., Simoes, M. J., and Cerri, P. S. (2015). Biology of bone tissue: structure, function, and factors that influence bone cells. Biomed Res. Int. 2015:421746. doi: 10.1155/2015/421746

Globus, R. K., Bikle, D. D., Halloran, B., and Morey-Holton, E. (2015). Skeletal response to dietary calcium in a rat model simulating weightlessness. J. Bone Miner. Res. 1986, 191-197.

Grano, M., Mori, G., Minielli, V., Barou, O., Colucci, S., Giannelli, G., et al. (2002). Rat hindlimb unloading by tail suspension reduces osteoblast differentiation, induces IL-6 secretion, and increases bone resorption in ex vivo cultures. Calcif. Tissue Int. 70, 176-185. doi: 10.1007/s00223-001-2034-6

Hornberger, T. A., Hunter, R. B., Kandarian, S. C., and Esser, K. A. (2001). Regulation of translation factors during hindlimb unloading and denervation of skeletal muscle in rats. Am. J. Physiol. Cell Physiol. 281, C179-C187. doi: 10.1152/ajpcell.2001.281.1.C179

Hurst, J. E., and Fitts, R. H. (2003). Hindlimb unloading-induced muscle atrophy and loss of function: protective effect of isometric exercise. J. Appl. Physiol. 95, 1405-1417. doi: 10.1152/japplphysiol.00516.2002

\section{FUNDING}

This work was supported by the National Research Foundation of Korea funded by the Ministry of Science, ICT and Future Planning (NRF-2013M3A9B6046417, Korea Mouse Phenotyping Project NRF-2013M3A9D5072550, 2013M3A9D5072560, 2017M3A9D5A01052447, and MEST 2011-030135) and by the Ministry of Education (NRF-2014R1A1A2058645).

Jadvar, H., Desai, B., and Conti, P. S. (2015). Sodium 18F-fluoride PET/CT of bone, joint, and other disorders. Semin. Nucl. Med. 45, 58-65. doi: 10.1053/j. semnuclmed.2014.07.008

Jang, J., Park, J., Chang, H., and Lim, K. (2016). 1-Carnitine supplement reduces skeletal muscle atrophy induced by prolonged hindlimb suspension in rats. Appl. Physiol. Nutr. Metab. 41, 1240-1247. doi: 10.1139/apnm-20160094

Jing, D., Cai, J., Wu, Y., Shen, G., Li, F., Xu, Q., et al. (2014). Pulsed electromagnetic fields partially preserve bone mass, microarchitecture, and strength by promoting bone formation in hindlimb-suspended rats. J. Bone Miner. Res. 29, 2250-2261. doi: 10.1002/jbmr.2260

Ju, Y. I., Sone, T., Ohnaru, K., Choi, H. J., Choi, K. A., and Fukunaga, M. (2013). Jump exercise during hindlimb unloading protect against the deterioration of trabecular bone microarchitecture in growing young rats. Springerplus 2:35. doi: 10.1186/2193-1801-2-35

Lang, T., LeBlanc, A., Evans, H., Lu, Y., Genant, H., and Yu, A. (2004). Cortical and trabecular bone mineral loss from the spine and hip in long-duration spaceflight. J. Bone Miner. Res. 19, 1006-1012. doi: 10.1359/JBMR.040307

Leblanc, A., Matsumoto, T., Jones, J., Shapiro, J., Lang, T., Shackelford, L., et al. (2013). Bisphosphonates as a supplement to exercise to protect bone during long-duration spaceflight. Osteoporos. Int. 24, 2105-2114. doi: 10.1007/s00198012-2243-z

Lloyd, S. A., Lang, C. H., Zhang, Y., Paul, E. M., Laufenberg, L. J., Lewis, G. S., et al. (2014). Interdependence of muscle atrophy and bone loss induced by mechanical unloading. J. Bone Miner. Res. 29, 1118-1130. doi: 10.1002/jbmr. 2113

Morey-Holton, E., Globus, R. K., Kaplansky, A., and Durnova, G. (2005). The hindlimb unloading rat model: literature overview, technique update and comparison with space flight data. Adv. Space Biol. Med. 10, 7-40. doi: 10.1016/ S1569-2574(05)10002-1

Morey-Holton, E. R., and Globus, R. K. (2002). Hindlimb unloading rodent model: technical aspects. J. Appl. Physiol. 92, 1367-1377. doi: 10.1152/japplphysiol. 00969.2001

Nakashima, T. (2015). [Frontiers in live bone imaging researches. Amazing function of osteocyte]. Clin. Calcium 25, 899-905.

Ohira, Y., Kawano, F., Wang, X. D., Sudoh, M., Iwashita, Y., Majima, H. J., et al. (2006). Irreversible morphological changes in leg bone following chronic gravitational unloading of growing rats. Life Sci. 79, 686-694. doi: 10.1016/j.lfs. 2006.02.022

Pan, Z., Yang, J., Guo, C., Shi, D., Shen, D., Zheng, Q., et al. (2008). Effects of hindlimb unloading on ex vivo growth and osteogenic/adipogenic potentials of bone marrow-derived mesenchymal stem cells in rats. Stem Cells Dev. 17, 795-804. doi: 10.1089/scd.2008.0254

Roer, R. D., and Dillaman, R. M. (1990). Bone growth and calcium balance during simulated weightlessness in the rat. J. Appl. Physiol. 68, 13-20. doi: 10.1152/ jappl.1990.68.1.13

Russo, C. R. (2009). The effects of exercise on bone. Basic concepts and implications for the prevention of fractures. Clin. Cases Miner. Bone Metab. 6, 223-228.

Sakata, T., Sakai, A., Tsurukami, H., Okimoto, N., Okazaki, Y., Ikeda, S., et al. (1999). Trabecular bone turnover and bone marrow cell development in tailsuspended mice. J. Bone Miner. Res. 14, 1596-1604. doi: 10.1359/jbmr.1999.14. 9.1596

Shirazi-Fard, Y., Metzger, C. E., Kwaczala, A. T., Judex, S., Bloomfield, S. A., and Hogan, H. A. (2014). Moderate intensity resistive exercise improves metaphyseal cancellous bone recovery following an initial disuse period, but 
does not mitigate decrements during a subsequent disuse period in adult rats. Bone 66, 296-305. doi: 10.1016/j.bone.2014.06.005

Song, J., Saeman, M. R., Baer, L. A., Cai, A. R., Wade, C. E., and Wolf, S. E. (2017). Exercise altered the skeletal muscle micrornas and gene expression profiles in burn rats with hindlimb unloading. J. Burn Care Res. 38, 11-19. doi: 10.1097/BCR.0000000000000444

Swift, J. M., Nilsson, M. I., Hogan, H. A., Sumner, L. R., and Bloomfield, S. A. (2010). Simulated resistance training during hindlimb unloading abolishes disuse bone loss and maintains muscle strength. J. Bone Miner. Res. 25, 564-574. doi: 10.1359/jbmr.090811

Tesch, P. A., Berg, H. E., Bring, D., Evans, H. J., and LeBlanc, A. D. (2005). Effects of 17-day spaceflight on knee extensor muscle function and size. Eur. J. Appl. Physiol. 93, 463-468. doi: 10.1007/s00421-004-1236-9

Tsika, R. W., Herrick, R. E., and Baldwin, K. M. (1987). Effect of anabolic steroids on skeletal muscle mass during hindlimb suspension. J. Appl. Physiol. 63, 2122-2127. doi: 10.1152/jappl.1987.63.5.2122

van der Eerden, B. C., Teti, A., and Zambuzzi, W. F. (2014). Bone, a dynamic and integrating tissue. Arch. Biochem. Biophys. 561, 1-2. doi: 10.1016/j.abb.2014. 08.012

Vico, L., Bourrin, S., Very, J. M., Radziszowska, M., Collet, P., and Alexandre, C. (1995). Bone changes in 6-mo-old rats after head-down suspension and a reambulation period. J. Appl. Physiol. 79, 1426-1433. doi: 10.1152/jappl.1995. 79.5.1426
Wang, J., Wang, F., Zhang, P., Liu, H., He, J., Zhang, C., et al. (2017). PGC-1alpha over-expression suppresses the skeletal muscle atrophy and myofiber-type composition during hindlimb unloading. Biosci. Biotechnol. Biochem. 81, 500-513. doi: 10.1080/09168451.2016.1254531

Warren, G. L., Stallone, J. L., Allen, M. R., and Bloomfield, S. A. (2004). Functional recovery of the plantarflexor muscle group after hindlimb unloading in the rat. E. J. Appl. Physiol. 93, 130-138. doi: 10.1007/s00421-004-1185-3

Westerlind, K. C., Fluckey, J. D., Gordon, S. E., Kraemer, W. J., Farrell, P. A., and Turner, R. T. (1998). Effect of resistance exercise training on cortical and cancellous bone in mature male rats. J. Appl. Physiol. 84, 459-464. doi: 10.1152/ jappl.1998.84.2.459

Conflict of Interest Statement: The authors declare that the research was conducted in the absence of any commercial or financial relationships that could be construed as a potential conflict of interest.

Copyright (c) 2018 Song, Cho, Lee, Lee and Song. This is an open-access article distributed under the terms of the Creative Commons Attribution License (CC BY). The use, distribution or reproduction in other forums is permitted, provided the original author(s) and the copyright owner(s) are credited and that the original publication in this journal is cited, in accordance with accepted academic practice. No use, distribution or reproduction is permitted which does not comply with these terms. 